\title{
Open and Laparoscopic Partial Nephrectomy: Comparison and Validation of Preoperative Scoring Systems, Including PADUA, RENAL, $A B C$ Nephrometric Scores and Perinephric Fat Evaluation with Mayo Adhesive Probability Score
}

\author{
Maxime Sempels' \\ Mohamed Ali Ben Chehida' \\ Paul Meunier ${ }^{2}$ \\ David Waltregny' \\ 'Department of Urology, University \\ Hospital of Liège, Liège, Belgium; \\ ${ }^{2}$ Department of diagnostic and interven- \\ tional Radiology, University Hospital of \\ Liège, Liège, Belgium
}

\begin{abstract}
Purpose: To evaluate potential associations between 4 nephrometric scoring systems, namely the PADUA (preoperative aspects and dimensions used for anatomical classification), RENAL (radius endophytic/exophytic nearness anterior posterior location), ABC (arterial-based complexity), and MAP (Mayo adhesive probability) scores and their individual components, with surgical and oncological outcomes of patients undergoing a partial nephrectomy.
\end{abstract}

Materials and Methods: A consecutive, monocentric cohort of partial nephrectomy patients was retrospectively analyzed. PADUA, RENAL, ABC and MAP nephrometry scores were determined from preoperative axial images. Unadjusted and adjusted associations between overall scores, individual components, surgical approach, complications and oncological outcomes were determined using univariate and multivariate logistic regressions.

Results: A total of 189 partial nephrectomies were performed in 181 patients, via an open or a laparoscopic approach. Among scoring systems, only the MAP classification, which assesses adherent perinephric fat, was associated with severe surgical complications as well as with operative time $(\mathrm{p}<0.05)$. Among all components of the PADUA and RENAL scores, only proximity of the tumor to the collecting system was associated with overall surgical complication rates, while the diameter of the tumor influenced the operative time $(p<0.05)$. The ABC score was not relevant. Male gender, antiplatelet therapy, and a laparoscopic approach were associated with higher overall surgical complication rates $(\mathrm{p}<0.05)$. The number of oncologic recurrences during follow-up was too low to run statistical analyses.

Conclusion: Nephrometry scores could be simplified to predict surgical complications after partial nephrectomy. In this framework, adherent perinephric fat seemed to be strongly associated with an increased risk of surgical complications.

Keywords: partial nephrectomy, scoring systems, perinephric fat, laparoscopy, complications

\section{Introduction}

Partial nephrectomy is the standard treatment for small renal masses, if technically feasible. ${ }^{1,2}$ As compared with radical surgery, nephron-sparing surgery may better preserve renal function, decrease the incidence of cardiovascular events, and offer similar oncological results with a higher quality of life. ${ }^{1-4}$ However, partial
Department of Urology,University Hospital of Liège, Avenue de l'hôpital, I, Liège, 4000 , Belgium

Tel +324366725 I

$\mathrm{Fax}+3243679506$

Email maxime.sempels@chuliege.be 
nephrectomy is potentially associated with severe periand postoperative complications, whether it is performed via an open or laparoscopic approach. ${ }^{5,6}$

An objective and rigorous preoperative evaluation is essential to confirm the technical feasibility, to predict the surgical complexity, and consequently to estimate the risks of complications, including bleeding and urinary leakage. ${ }^{7}$ Moreover, a standardized evaluation using defined parameters would be helpful to compare series from different centers with various surgical approaches. In this respect, patient's characteristics as well as the features of their tumor(s) should be integrated into this evaluation.

Patient's characteristics include co-morbidities and medications, as well as potentially relevant anatomical elements, such as attributes of perirenal fat. Indeed, the presence of adherent perinephric fat has been suspected to increase perioperative and post-surgical morbidity, to be associated with unfavorable tumor histology, and to bear an impact on oncological outcomes. ${ }^{8,9}$ The MAP score (Mayo Adhesive Probability score) has been proposed to evaluate the extent of adherent perinephric fat. ${ }^{10}$

Features of the tumor are grouped under morphometry scores, amongst which the RENAL (Radius Endophytic/ exophytic Nearness Anterior posterior Location) and PADUA (Preoperative Aspects and Dimensions Used for Anatomical classification) scores are the most widely known. ${ }^{11,12}$ However, these scores are not routinely used in clinical practice, and their place is not well defined yet. ${ }^{13-16}$ As a matter of fact, these scores could be more effective and simplified. For instance, a study performed in Canada has compared these tumor scoring systems (RENAL and PADUA), and has shown that PADUA scoring was associated with surgical and overall complications, but among all components of the scoring system, only a large diameter of the tumor and its endophytic nature were significantly associated with a higher risk of complications. ${ }^{17}$ In this context, several new scores have been developed, with the aim of being easier for use in routine clinical practice. The following list is not exhaustive: the C-index score, the DAP nephrometry score, the Renal tumor invasion index, the Contact surface area score, the Resected and ischemized volume, the Zonal nephro score, the Surgical approach renal ranking, the $\mathrm{ABC}$ score, and the Renal pelvis score. ${ }^{7,18}$ Among all these, according to our opinion, the ABC score (arterialbased complexity) appears to be rather simple and relevant. ${ }^{19}$
In this study, we have sought to evaluate a number of nephrometry scoring systems, as well as their individual components, and to evaluate potential associations between the overall scores - and individual components of these scores - with surgical and oncological outcomes. We have arbitrarily chosen to evaluate RENAL, PADUA, MAP and $\mathrm{ABC}$ scores. We also aimed at investigating potential associations between the surgical approach used, co-morbidities and medications of the patients, with surgical and oncological outcomes.

\section{Materials and Methods}

A consecutive cohort of patients who had undergone a partial nephrectomy in our center between 2006 and 2015 was retrospectively analyzed. Two surgical approaches were used: open surgery (lombotomy) and laparoscopic surgery (trans-peritoneal). The choice for the surgical approach was made according to the surgeon preference. Cases considered (subjectively) as feasible in a laparoscopic approach were referred to a single laparoscopic surgeon.

We have collected the characteristics of the patients and their tumor(s), as well as the surgical outcomes.

Characteristics of the patients included age, gender, body mass index (BMI), co-morbidities according to the Charlson score, ${ }^{20}$ medications, preoperative renal function, postoperative renal function, renal vasculature and perinephric fat aspect.

Features of the tumors included size, endophycity, proximity to the collecting system, location, contact with the hilum, and relationship to the vasculature.

For each patient, the most recent axial imaging (CTscan or MRI) was reviewed by two physicians, who were not independent, and who reached a consensus for each case. Scores for all 4 nephrometric systems (RENAL, PADUA, ABC and MAP) were calculated as defined in the original articles. Furthermore, each component of RENAL, PADUA and MAP scores were recorded individually.

The RENAL score included Tumor size (R), exophytic/ endophytic (E), nearness to the collecting system/renal sinus $(\mathrm{N})$, anterior/posterior location $(\mathrm{A})$, location relative to polar line (L). ${ }^{11}$

The PADUA score encompassed tumor diameter, location relative to polar lines, anterior/posterior location, medial/lateral location, collecting system relationship, renal sinus relationship, and exophytic/endophytic extent. $^{12}$ 
The RENAL and PADUA score included a number of components that were similar; however, they differed in their definition for some components (eg, location relative to polar lines). These scores were stratified in three subgroups: low, moderate and high risk.

The MAP score included posterior perinephric fat thickness and perinephric fat stranding. ${ }^{10}$ We have arbitrarily defined two subgroups (low risk for scores 0-1-2 (/5); high risk for scores 3-4-5 (/5)).

In the $\mathrm{ABC}$ score, renal masses were categorized by the order of vessels needed to be transected/dissected during partial nephrectomy. Scores of 1, 2, 3S, or 3H were assigned to tumors requiring transection of interlobular and arcuate arteries, interlobar arteries, segmental arteries, or in close proximity of the renal hilum, respectively, during partial nephrectomy. ${ }^{19}$ Two subgroups were arbitrarily defined (low risk for scores 1-2; high risk for scores 3S-3H).

In addition, vascular complexity was defined by the presence of a renal polar artery, a renal hilar artery, and/or an early bifurcation of the renal artery. ${ }^{7}$ The perinephric fat density was also assessed (by Hounsfield units (HU)) and two subgroups were arbitrarily defined ( $\leq 90 \mathrm{HU} ; \geq 90 \mathrm{HU})$.

Perioperative outcomes were abstracted from surgical reports, discharge summaries, follow-up visits, postoperative imaging and laboratory investigations.

Surgical outcomes included operative time, estimated blood loss, warm ischemia time, hospital stay, postoperative renal function and complications. Operative time was defined with a cut-off value of 120 minutes ( $<$ or $>$ to 120 minutes). Warm ischemia was defined according to the need to clamp a renal artery, and also according to the time of ischemia (cut-off value of 15 minutes). Postoperative renal function was estimated with the glomerular filtration rate (GFR) at 1 month and 1 year. Complications within 30 days were divided into surgical and medical, classified according to the Clavien system, ${ }^{21}$ and defined following EAU guidelines. The follow-up schedule was ensured according to EAU guidelines. It included clinical examination and computed tomography ( 2 times a year during 2 years, once a year until fifth year and then 1 every two years thereafter).

Oncological outcomes included tumor histology, Fuhrman's grade, margin status, TNM classification, local recurrence, metastasis, cancer-specific survival, secondary treatments and overall survival. Local recurrence is defined by a recurrence of tumoral tissue with the same histology than the primary tumor, located in the renal or perirenal area of the initially pathological kidney. A tumor from another histology or depending on another area of the kidney parenchyma is considered as a new primary tumor. Systemic recurrence is defined by a recurrence of tumoral tissue in lymphatic nodes or in any other organ of the body.

Statistical analysis: A univariable log binomial regression was performed between surgical approach, patient's and tumor's characteristics (including RENAL score, PADUA score, MAP score, ABC score, perinephric density and vascular complexity), with perioperative surgical outcomes. In addition, associations between each component of each scoring system and outcomes were evaluated.

Significant variables in the univariable analyses were included in a multivariable regression and a stepwise procedure was applied. Results were expressed as numbers, frequency, odds ratio (OR $(95 \% \mathrm{CI})$ ) and hazard ratio (HR $(95 \% \mathrm{CI}))$. Results were significant when $\mathrm{p}$-value was $<0.05$. The software used was SAS 9.4 version and R 3.2.2 version of Windows.

\section{Results}

One hundred and eighty-nine partial nephrectomies were performed in 181 patients. For patients with more than one tumour, partial nephrectomies were always asynchronous. Imaging was available for 157 patients. Charlson score was more than 2 in 73 patients (38\%), and ASA score was 1 or 2 in $93 \%$ of the patients. Median size of the tumors was 3 centimeters $(\min 0.8 ; \max 12)$. One hundred and forty-four (77\%) nephrectomies were performed by open surgery, and 45 (23\%) performed by a laparoscopic approach. Median RENAL, PADUA, and ABC scores were 6, 8, and 2, respectively (Table 1). These scores seemed to be homogenous between the open and the laparoscopic groups, but the proportion of high-risk cases (higher nephrometry scores) was higher in the open group (Figure 1). Median MAP score was 2 , and was completely homogenous between the open and the laparoscopic groups.

Median operative time was 120 minutes. Arterial clamping was performed in $58 \%$ of the patients, and median warm ischemia time for these patients was 12 minutes. Median blood loss was 2,1g/dl of haemoglobin. Median hospital stay was 7 days. Twenty-two (12\%) patients presented a significant loss of renal function (more than a 10\% GFR decrease). There were no differences in these perioperative surgical between the laparoscopic and open groups.

Among the 189 patients, 39 (21\%) experienced one or more surgical complication(s); 24 (13\%) were severe (Clavien 3-4) (Table 2). Surgical complications included 
Table I Overall Characteristics of the Population

\begin{tabular}{|c|c|}
\hline $\begin{array}{l}\text { Patient, Tumor and } \\
\text { Operative Characteristics }\end{array}$ & \\
\hline Cohort & $\begin{array}{l}\text { I89 partial nephrectomies on } 18 \mathrm{I} \text { patients } \\
\text { ( } 4 \text { nephrectomies on } 1 \text { patient, and } 2 \\
\text { nephrectomies on } 5 \text { patients) }\end{array}$ \\
\hline Sexe ratio & I.5 male /I female \\
\hline Age & Median: 59; (min-max: $21-85)$ \\
\hline BMI & Median: 26; (min-max: 17-42) \\
\hline Tumor diameter & $\begin{array}{l}\text { Median: } 3 \mathrm{~cm} \text {; }(\min -\max : 0.8-12 \mathrm{~cm}) ;(P 25- \\
\text { P75: } 2-4 \mathrm{~cm})\end{array}$ \\
\hline Renal Score (4-12) & $\begin{array}{l}\text { Median: } 6 \\
\text { Low risk: } 58 \text { patients/Moderate risk: } 82 \\
\text { patients/High risk: } 17 \text { patients }\end{array}$ \\
\hline Padua Score (6-14) & $\begin{array}{l}\text { Median: } 8 \\
\text { Low risk: } 47 \text { patients/Moderate risk: } 56 \\
\text { patients/High risk: } 54 \text { patients }\end{array}$ \\
\hline ABC Score $(1-3)$ & $\begin{array}{l}\text { Median: } 2 \\
\text { Low risk: } 23 \text { patients/Moderate risk: } 87 \\
\text { patients/High risk: } 48 \text { patients }\end{array}$ \\
\hline MAP Score $(I-5)$ & $\begin{array}{l}\text { Median: } 2 \\
\text { Low risk: } 92 \text { patients/High risk: } 65 \text { patients }\end{array}$ \\
\hline Open surgery & I44 patients (77\%) \\
\hline Laparoscopic surgery & 45 patients $(23 \%)$ \\
\hline Operative time & Median: 120 minutes $(\min 60 ; \max 252)$ \\
\hline Arterial clamping & 107 patients $(57 \%)$ \\
\hline Warm ischemia time & Median: 12 minutes $(\min 5 ; \max 30)$ \\
\hline Blood loss & $\begin{array}{l}\text { Median: } 2, \mid \mathrm{g} / \mathrm{dl} \text { of haemoglobin }(\min 0.1 \text {; } \\
\max 6.5 \text { ). }\end{array}$ \\
\hline Hospital stay & Median: 7 days $(\min 3 ; \max 26)$. \\
\hline Pathological findings & $\begin{array}{l}\text { I53 (8I\%) malignant tumors (renal } \\
\text { carcinoma); with I68 (89\%) PTI } \\
36 \text { (19\%) benign tumors (oncocytoma or } \\
\text { angiomyolipoma) }\end{array}$ \\
\hline
\end{tabular}

retroperitoneal hematoma, pseudoaneurysm, urine leakage, bleeding necessitating transfusion, perioperative kidney haemorrhage necessitating immediate conversion to radical nephrectomy, urinary tract infection, perinephric abscess, wound infection, splenectomy, secondary conversion to radical nephrectomy.

Twenty-three (12\%) patients had one or more medical complication(s). Two (1\%) were severe (Table 2). Medical complications included cardiovascular (arrhythmia, vasopressive support), pulmonary (pneumonia, respiratory
A
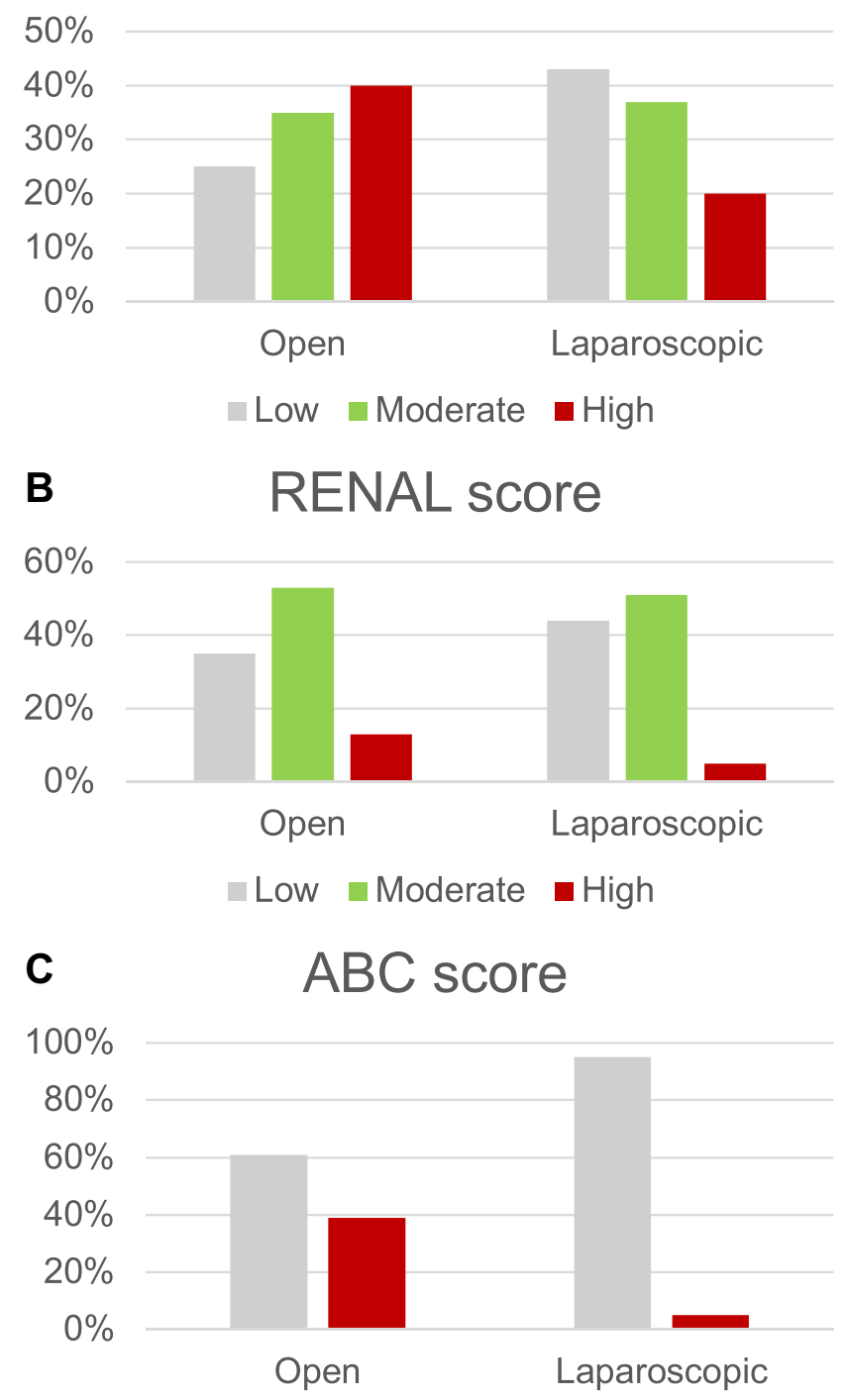

$1 \& 2=3 S \& 3 \mathrm{H}$

Figure I Proportion of low, moderate or high risk cases in the open and laparoscopic groups, according to PADUA, RENAL and ABC scores. (A) Proportion of low, moderate or high risk cases in the open and laparoscopic groups, according to PADUA score. (B) Proportion of low, moderate or high risk cases in the open and laparoscopic groups, according to RENAL score. (C) Proportion of low, moderate or high risk cases in the open and laparoscopic groups, according to $A B C$ score.

failure), gastrointestinal (prolonged ileus, pancreatitis) or infectious (urinary tract infection) events.

Eighty-one percent of tumors were malignant, of which $89 \%$ were staged pT1. Margins were positive in 11 patients (7\%) (Table 1). Median follow-up was 60 months $(\min 3 ; \max 156)$, and $6(4.5 \%)$ patients experienced a recurrence (local in 2 patients and metastatic in 4 patients). Only one of these 6 patients had positive surgical margin at partial nephrectomy. The 2 patients with a local recurrence were treated either by surgery or by radiation 
Table 2 Description of Global and Severe Complications (According to Clavien Classification)

\begin{tabular}{|c|c|c|c|}
\hline Surgical Complications & Global (n; \%) = Any Clavien & Severe $(n ; \%)=$ Clavien 3-4 & Relative Treatments \\
\hline Total & $39(21 \%)$ & $24(13 \%)$ & \\
\hline Retroperitoneal hematoma & 14 (7\%) & $8(4 \%)$ & $\begin{array}{l}\text { Surveillance ( } 6 \text { patients) } \\
\text { Selective embolisation ( } 8 \text { patients) }\end{array}$ \\
\hline Transfusion & $9(5 \%)$ & - & \\
\hline Urine leakage (fistula) & $5(3 \%)$ & $4(2 \%)$ & $\begin{array}{l}\text { Surveillance (I patient) } \\
\text { JJ stent ( } 4 \text { patients) }\end{array}$ \\
\hline Pseudoaneurysm & $5(3 \%)$ & $5(3 \%)$ & Selective embolisation \\
\hline Perioperative haemorrhage & $8(4 \%)$ & $8(4 \%)$ & Immediate radical nephrectomy \\
\hline Urinary infection & $3(3 \%)$ & - & Antibiotics \\
\hline Wound infection & $3(3 \%)$ & - & Wound opened at bedside \\
\hline Perinephric abscess & I $(0,5 \%)$ & - & Antibiotics \\
\hline Secondary nephrectomy & $2(1 \%)$ & $2(1 \%)$ & \\
\hline Spleen traumatism & I $(0,5 \%)$ & I $(0,5 \%)$ & Immediate splenectomy \\
\hline \multicolumn{4}{|l|}{ Medical Complications } \\
\hline Total & $23(12 \%)$ & $2(1 \%)$ & \\
\hline Prolonged bowel ileus & $5(3 \%)$ & - & Diet and gastroprokinetics \\
\hline Bronchopneumonia & $4(2 \%)$ & - & Antibiotics and physiotherapy \\
\hline Lung atelectasis & $4(2 \%)$ & - & Physiotherapy \\
\hline Indeterminate fever & $3(1,5 \%)$ & - & Antibiotics \\
\hline Arythmia & $2(1 \%)$ & - & Antiarythmic drugs \\
\hline Vasopressive support & I $(0,5 \%)$ & I $(0,5 \%)$ & Intensive care, vasopressive drugs \\
\hline Respiratory failure & I $(0,5 \%)$ & I $(0,5 \%)$ & Intensive care, oxygen therapy \\
\hline Prostatitis & I $(0,5 \%)$ & - & Antibiotics \\
\hline Pancreatitis & I $(0,5 \%)$ & - & Medical treatment \\
\hline
\end{tabular}

therapy, with a recurrence-free status during the following years. Cancer-specific mortality was 3\% (4 patients), with a median time to death of 24 months.

Operative time was influenced by several elements: MAP score $(p=0.0001)$, tumor diameter $(p=0.0002)$, nearness to the collecting system $(\mathrm{p}=0.0025)$, total PADUA score $(p=0.0054)$, vascular complexity $(p=0.011)$, collecting system relationship $(\mathrm{p}=0.014)$, total RENAL score $(\mathrm{p}=0.014)$, renal sinus relationship $(\mathrm{p}=0.024), \mathrm{ABC}$ score $(p=0.047)$ on univariate analyses; and MAP score and a tumor diameter $>4 \mathrm{~cm}(\mathrm{p}=0.0001$ and $\mathrm{p}=0.0002$, respectively) on multivariate analyses (Table 3).

The need for arterial clamping is only associated in our cohort with tumor diameter $(\mathrm{p}=0.0002)$. Warm ischemia time was associated with the following items: nearness to the collecting system $(p=0.0001)$, total RENAL score $(p=0.011)$, relationship with the collecting system $(\mathrm{p}=0.012)$, laparoscopic approach $(p=0.018)$, tumor diameter $(p=0.031), A B C$ score $(\mathrm{p}=0.038)$ on univariate analyses; and only tumor diameter $(\mathrm{p}=0.0002)$ on multivariate analyses (Table 3 ).

Table 3 Significant Associations ( $p$-value<0,05) Between Patient, Operative, and Tumour Characteristics with Operative Time. Multivariate Analyses

\begin{tabular}{|l|l|l|l|l|}
\hline Outcome & Predictive Factors After Multivariate Analyses & OR & $\mathbf{9 5 \%}$ IC & p-value \\
\hline Operative time $>$ 120min & High MAP score & 4.564 & $2.19-9.471$ & 0.0001 \\
& Tumor diameter $>4 \mathrm{~cm}$ & 5.027 & $2.15-11.7$ & 0.0002 \\
\hline Need of warm ischemia & Tumor diameter $>4 \mathrm{~cm}$ & 4.533 & $2.04-10.07$ & 0.0002 \\
\hline
\end{tabular}


No factor influenced blood loss in our series; the ABC score was at the limit of significance $(\mathrm{p}=0.05)$.

Surgical complications were significantly associated with preoperative renal function $(\mathrm{p}=0.0039)$, male gender $(p=0.011)$, antiplatelet therapy $(p=0.011)$, nearness to the collecting system $(\mathrm{p}=0.013)$, laparoscopic approach $(\mathrm{p}=0.018)$, and the MAP score $(\mathrm{p}=0.022)$ on univariate analyses (Table 4); and with the nearness to the collecting system $(\mathrm{p}=0.0006)$, male gender $(\mathrm{p}=0.0028)$, antiplatelet therapy $(\mathrm{p}=0.0042)$, and a laparoscopic approach $(\mathrm{p}=0.0062)$ on multivariate analyses (Table 5).

Severe surgical complications were associated with MAP score $(\mathrm{p}=0.0014)$, laparoscopic approach $(\mathrm{p}=0.0090)$, preoperative renal function $(\mathrm{p}=0.012)$, on univariate analyses (Table 4); and with the MAP score and with a laparoscopic approach $(\mathrm{p}=0.0020$ and $\mathrm{p}=0.0372$, respectively) on multivariate analyses (Table 5).

Regarding medical complications, only a lower preoperative renal function was associated with a higher risk of global medical events (Table 6). A lower preoperative renal function was by the way the only predictor for a significant loss of renal function. The number of severe medical complications was too low to draw conclusions.

Length of hospital stay was significantly influenced by age and tumor diameter $(\mathrm{p}=0.036$ and 0.0036$)$ on multivariate analysis.

The number of oncological events during follow-up was also too low to carry out statistical analyses. Regarding cases with positive margins, the only risk factor highlighted was a pathological stage pT3 $(\mathrm{p}=0.035)$.

\section{Discussion}

Surgical and oncological results in our series were globally similar to those found in the literature. ${ }^{1,5,6}$

The number of severe surgical complications is quite high but a potential reason is that we have included some operative complications, like "splenectomy" or "conversion to radical nephrectomy", into our global surgical complications. These complications could be considered as modifications of the operative plan due to technical

Table 4 Significant Associations ( $\mathrm{p}$-value $<0,05$ ) Between Patient, Operative, and Tumour Characteristics with Total and Severe Surgical Complications. Univariate Analyses

\begin{tabular}{|l|l|l|l|l|}
\hline Outcome & Predictive Factors After Univariate Analyses & OR & 95\% Cl & p-value \\
\hline \multicolumn{2}{|l|}{ Surgical complications } & \multicolumn{3}{l|}{} \\
\hline \multirow{2}{*}{ Any Clavien } & Preoperative impaired renal function & 3.48 & $1.49-8.11$ & 0.0039 \\
& Male gender & 0.34 & $0.15-0.78$ & 0.011 \\
& Antiplatelet agents taking & 2,54 & $1.23-5.21$ & 0.011 \\
& Nearness to the collecting system & 4.67 & $1.49-14.6$ & 0.013 \\
& Laparoscopic approach & 2.5 & $1.17-5.34$ & 0.018 \\
\hline \multirow{2}{*}{ Clavien 3-4 } & MAP score & 2.48 & $1.14-5.38$ & 0.022 \\
& High MAP score & 5.68 & $1.96-16.46$ & 0.0014 \\
& Laparoscopic approach & 3.26 & $1.34-7.92$ & 0.0090 \\
& Preoperative impaired renal function & 3.43 & $1.31-8.99$ & 0.0121 \\
\hline
\end{tabular}

Table 5 Significant Associations ( $p$-value<0,05) Between Patient, Operative, and Tumour Characteristics with Total and Severe Surgical Complications. Multivariate Analyses

\begin{tabular}{|l|l|l|l|l|}
\hline Outcome & Predictive Factors After Multivariate Analyses & OR & $\mathbf{9 5 \%} \mathbf{C l}$ & $\mathbf{p - v a l u e ~}$ \\
\hline \multicolumn{2}{|l|}{ Surgical complications } & \multicolumn{4}{l|}{} \\
\hline \multirow{2}{*}{ Any Clavien } & Nearness to the collecting system & 7.975 & $2.22-28.63$ & 0.0006 \\
& Male gender & 0.198 & $0.06-0.57$ & 0.0028 \\
& Antiplatelet therapy & 3.614 & $1.49-8.72$ & 0.0042 \\
& Laparoscopic approach & 3.826 & $1.46-10.00$ & 0.0062 \\
\hline Clavien 3-4 & High MAP score & 5.443 & $1.85-15.9$ & 0.0020 \\
& Laparoscopic approach & 2.864 & $1.06-7.70$ & 0.0372 \\
\hline
\end{tabular}


Table 6 Significant Associations ( $p$-value<0,05) Between Patient, Operative, and Tumour Characteristics with Total and Severe Medical Complications. Univariate Analyses

\begin{tabular}{|l|l|l|l|l|}
\hline Outcome & Predictive Factors After Univariate Analyses & OR & 95\% IC & P-value \\
\hline Medical complications & \multicolumn{3}{|l|}{} & \\
\hline $\begin{array}{l}\text { Any Clavien } \\
\text { Clavien 3-4 }\end{array}$ & $\begin{array}{l}\text { Preoperative impaired renal function } \\
-\end{array}$ & 4.69 & $1.80-12.25$ & 0.0016 \\
\hline
\end{tabular}

difficulties and not as real surgical complications. But we decided to consider these complications because they are potential reflection of surgical complexity and it was then interesting to include them in our correlation analysis with nephrometric scores and adherent perinephric fat.

The number of tumor recurrences during follow-up was too low to correlate tumor features - including nephrometry scores and their individual components - as well as patient characteristics, with oncologic outcomes.

Nevertheless, our study enabled to identify interesting predictive factors of surgical outcomes, including complications.

A number of patient features were associated with a higher risk of postoperative complications. Male gender and antiplatelet therapy were significantly associated with a higher risk of surgical complications. These data emphasize on the importance of a careful management of platelet aggregation inhibitors in the perioperative period.

Our data also largely confirmed the potential impact of assessing perinephric fat. ${ }^{22,23}$ Indeed, in our study, a high MAP score was strongly associated with an increased risk of severe surgical complications and with prolonged operative time. Furthermore, as described in the original article, the MAP score was in our experience easy to use, and may therefore become a clinically meaningful tool for preoperative evaluation. ${ }^{10}$ However, it could be possible that experience and skills of the surgeon are likely to overcome difficulty during perinephric fat dissection, like suggested in the recent literature. ${ }^{24}$

Some tumor features, in particular a couple of individual components of global nephrometric scores, were also associated with several peri- and postoperative outcomes. Indeed, following adjustments, no association was found between overall scores (RENAL, PADUA and ABC scores) and surgical outcomes. However, among all individual components of the PADUA and RENAL scores, the nearness of the tumor to the collecting system was significantly associated with overall surgical complications and the tumor diameter significantly influenced operative time $(\mathrm{p}<0.05)$. Furthermore, the diameter of the tumor was associated with the rate of renal artery clamping. Actually, the nearness of the tumor to the collecting system and its diameter may represent the 2 most significant measures assessed through morphometric evaluations. The $\mathrm{ABC}$ score seemed to be interesting, logical, and easy to use, and authors demonstrated correlations between this score and perioperative morbidity (ischemia time, estimated blood loss, urinary fistula formation), but these associations were not demonstrated in our study and this score was therefore not relevant.

When studying and comparing scores in the literature, there are conflicting results and there is therefore no one better than the others. ${ }^{7,18}$

Some authors recommend modifications on the available scores. Indeed, Salah et al recently described the newly modified nephrometry score, which includes incorporation of hilar position of the mass as a numerical score as well as incorporation of renal pelvis score to RENAL and give higher points to certain variables according to their importance in describing the complexity of the mass. This newly modified nephrometry score was associated in their study with better prediction of outcome of partial nephrectomy when compared to R.E. N.A.L. ${ }^{25}$ Some other studies already suggest using simplified scores. Desantis et al suggest that a score incorporating tumour size and some measure of the tumour's depth/contact with the renal parenchyma can likely perform at least as well as RENAL or PADUA systems. ${ }^{17}$ Corradi et al demonstrated that tumor size and depth are important characteristics for predicting robotic PN outcomes. They concluded these features could be used individually as a simplified way to report tumor features for research and patient counseling purposes. ${ }^{26}$ Ficarra et al proposed recently the simplified PADUA REnal score. This new SPARE score is comprised of only four variables instead of the original six and its accuracy to predict overall complications is similar to that of the original PADUA score. ${ }^{27,28}$ 
At last, we can also discuss other imaging tools to assess tumour complexity such as three-dimensional (3D) imaging. ${ }^{29,30}$ Porpiglia et al proposed recently a $3 \mathrm{D}$ virtual model based on PADUA and RENAL nephrometry scores, and showed that 3D virtual models were more precise than 2D standard imaging in evaluating the surgical complexity of renal masses and were better in predicting postoperative complications. ${ }^{31}$ Because of technical considerations, and in order to simplify our study, we have not integrated these 3D models in this study, but these new 3D imaging tools are very interesting and need to be evaluated in other series.

Overall, the RENAL and PADUA scores may be too complex and of limited usefulness in routine clinical practice. Ultimately, nephrometry scores could be simplified, using only two tumor features, namely the nearness of the tumor to the collecting system and its diameter, and this simplified preoperative evaluation could also integrate the risk of adherent perinephric fat.

Finally, regarding the surgical approach, laparoscopic surgery in this study was clearly associated with a higher risk to develop severe surgical complications, even though the surgical cases seemed simpler. All laparoscopic partial nephrectomies were performed by one single surgeon in our team. One may hypothesize that the surgical experience had not yet reached beyond the learning curve to explain a higher rate of surgical complications. It may be suggested that such a laparoscopic approach should be proposed only in high-volume centers, by experienced hands, and should be avoided in complex cases.

\section{Study Limitations}

At first, the retrospective nature of the study limits the strength of the results.

Moreover, this study includes both open (77\%) and laparoscopic procedures, also performed by different surgeons with different surgical expertise.

Finally, imaging was available for 159 cases and our statistical analysis could then only be made out of these 159 cases and not of the whole cohort.

\section{Conclusions}

Nephrometry scores could be simplified to predict surgical complications after partial nephrectomy. Indeed, only the diameter of the tumor and the nearness of the collecting system seemed to be useful. The ABC score was not relevant in our study. Adherent perinephric fat, evaluated by the MAP score, seemed to be strongly associated with surgical complications and warrants further investigations. The laparoscopic approach was a risk factor for surgical complications.

\section{Abbreviations}

RENAL, Radius - Endophytic/exophytic - Nearness Anterior posterior - Location; PADUA, Preoperative Aspects and Dimensions Used for Anatomical classification; ABC Score, Arterial-Based Complexity Score; MAP Score, Mayo Adhesive Probability Score.

\section{Ethics Approval}

For this retrospective study, according to Belgian laws, the ethics committee of the University Hospital of Liège and Faculty of Medicine of Liège did not consider necessary an ethics approval or a patient consent. Because of the retrospective and observational character of this study, the ethics committee of the University Hospital of Liège and Faculty of Medicine of Liège did not consider necessary an ethics approval or a patient consent. We confirm that this work was made in accordance with the ethical principles presented in the latest version of the declaration of Helsinki. Patient data confidentiality was absolutely respected.

\section{Disclosure}

The authors report no conflicts of interest in this work.

\section{References}

1. Lesage K, Joniau S, Fransis K, Van Poppel H. Comparison between open partial and radical nephrectomy for renal tumours: perioperative outcome and health-related quality of life. Eur Urol. 2007;51 (3):614-620. doi:10.1016/j.eururo.2006.10.040

2. Van Poppel H, Da Pozzo L, Albrecht W. A prospective, randomised EORTC intergroup Phase 3 study comparing the oncologic outcome of elective nephron-sparing surgery and radical nephrectomy for low-stage renal cell carcinoma. Eur Urol. 2011;59(4):543-552. doi:10.1016/j.eururo.2010.12.013

3. Clark ATD, Breau RH, Morash C. Preservation of renal function following partial or radical nephrectomy using 24-hour creatinine clearance. Eur Urol. 2008;54(1):143-149. doi:10.1016/j. eururo.2008.03.037

4. Reix B, Bernhard JC, Patard JJ, et al. Overall survival and oncological outcomes after partial nephrectomy and radical nephrectomy for cT2a renal tumors: a collaborative international study from the French kidney cancer research network UroCCR. Prog Urol. 2018;28 (3):146-155. doi:10.1016/j.purol.2017.12.004

5. Gill IS, Kavoussi LR, Lane BR, Blute ML, Babineau D, Colombo JR. Comparison of 1800 laparoscopic and open partial nephrectomies for single renal tumors. $J$ Urol. 2007;178(1):41-46. doi:10.1016/j. juro.2007.03.038

6. Vittori G. Open versus robotic-assisted partial nephrectomy: a multicenter comparison study of peri-operative outcomes and complications. World J Urol. 2014;32(1):287-293. doi:10.1007/ s00345-013-1136-x 
7. Klatte T, Ficarra V, Gratzke C, Kaouk J, Kutikov A, Macchi A. A literature review of renal surgical anatomy and surgical strategies for partial nephrectomy. Eur Urol. 2015;68(6):980-992. doi:10.1016/ j.eururo.2015.04.010

8. Kocher Neil J, Kunchala S, Reynolds C, Lehman E, Nie S, Raman JD. Adherent perinephric fat at minimally invasive partial nephrectomy is associated with adverse peri-operative outcomes and malignant renal histology. BJU Int. 2016;117(4):636-641. doi:10.1111/bju. 13378

9. Thiel DD, Davidiuk AJ, Meschia C, et al. Mayo Adhesive probability score is associated with localized renal cell carcinoma progression-free survival. Urology. 2016;89:54-60. doi:10.1016/j. urology.2015.10.034

10. Davidiuk Andrew J, Parker Alexander S, Thomas Colleen S, et al. Mayo adhesive probability score: an accurate image-based scoring system to predict adherent perinephric fat in partial nephrectomy. Eur Urol. 2014;66(6):1165-1171. doi:10.1016/j.eururo.2014.08.054

11. Kutikov A, Uzzo RG. The R.E.N.A.L nephrometry score: a comprehensive standardized system for quantitating renal tumor size, location and depth. J Urol. 2009;182(3):844-853. doi:10.1016/j. juro.2009.05.035

12. Ficarra V, Novara G, Secco S, et al. Preoperative aspects and dimensions used for an anatomical (PADUA) classification of renal tumours in patients who are candidates for nephron-sparing surgery. Eur Urol. 2009;56(5):786-793. doi:10.1016/j.eururo.2009.07.040

13. Canter D, Kutikov A, Manley B. Utility of the R.E.N.A. L. nephrometry scoring system in objectifying treatment decision-making of the enhancing renal mass. Urology. 2011;78 (5):1089-1094. doi:10.1016/j.urology.2011.04.035

14. Lavallée LT, Desantis D, Kamal F. The association between renal tumor scoring systems and ischemia time during open partial nephrectomy. Can Urol Assoc J. 2012;6(3):1-8. doi:10.5489/ cuaj. 11202

15. Stroup SP, Palazzi K, Kopp RP. RENAL nephrometry score is associated with operative approach for partial nephrectomy and urine leak. Urology. 2012;80(1):151-156. doi:10.1016/j.urology.20 12.04.026

16. Hew MN, Baseskioglu B, Barwari K. Critical appraisal of the PADUA classification and assessment of the R.E.N.A. L. nephrometry score in patients undergoing partial nephrectomy. J Urol. 2011;186(1):42-46. doi:10.1016/j.juro.2011.03.020

17. Desantis D, Lavallée Luke T, Witiuk K, et al. The association between renal tumour scoring system components and complications of partial nephrectomy. Can Urol Assoc J. 2015;9(1-2):39-45. doi: $10.5489 /$ cuaj. 2303

18. Hou W, Yan W, Ji Z. Anatomic features involved in technical complexity of partial nephrectomy. Urology. 2015;85(1):1-7. doi:10.1016/j.urology.2014.10.009

19. Spaliviero M, Poon BY, Karlo Christoph A, et al. An Arterial Based Complexity (ABC) scoring system to assess the morbidity profile of partial nephrectomy. Eur Urol. 2016;69(1):72-79. doi:10.1016/j. eururo.2015.08.008
20. Charlson ME, Pompei P, Ales KL. A new method of classifying prognostic comorbidity in longitudinal studies: development and validation. J Chronic Dis. 1987;40(5):373-383. doi:10.1016/00219681(87)90171-8

21. Dindo D, Demartines N, Clavien PA. Classification of surgical complications: a new proposal with evaluation in a cohort of 6336 patients and results of a survey. Ann Surg. 2004;240(2):205-213. doi:10.1097/01.sla.0000133083.54934.ae

22. Bier S, Aufderklamm S, Todenhöfer T, et al. Prediction of postoperative risks in laparoscopic partial nephrectomy using RENAL, Mayo adhesive probability and renal pelvic score. Anticancer Res. 2017;37(3):1369-1373.

23. Ishiyama R, Kondo T, Takagi T, et al. Impact of the Mayo adhesive probability score on the complexity of robo-assisted partial nephrectomy. $J$ Endourol. 2018;32(10):928-933. doi:10.1089/ end.2017.0779

24. Di Maida F, Vittori G, Campi R, et al. Clinical predictors and significance of adherent perinephric fat assessed with Mayo Adhesive Probability (MAP) score and Perinephric Fat Surface Density (PnFSD) at the time of partial nephrectomy for localized renal mass. A single high-volume referral center experience. Minerva Urol Nefrol. 2020

25. Salah M, Elsheemy M, Ghoneima M, et al. Modified R.E.N.A.L nephrometry score for predicting the outcome following partial nephrectomy. Afr J Urol. 2020;26(45). doi:10.1186/s12301-02000056-3.

26. Corradi R, Vertosick E, Nguyen D, et al. Nephrometry scores and perioperative outcomes following robotic partial nephrectomy. Int Braz J Urol. 2017;43(6):1075-1083. doi:10.1590/s1677-5538. ibju.2016.0571

27. Ficarra V, Porpiglia F, Crestani A, et al. The Simplified PADUA REnal (SPARE) nephrometry system: a novel classification of parenchymal renal tumours suitable for partial nephrectomy. BJU Int. 2019;124(4):621-628. doi:10.1111/bju.14772

28. Huang $\mathrm{CP}$, Chang $\mathrm{CH}, \mathrm{Wu} \mathrm{HC}$, et al. External validation of the Simplified PADUA REnal (SPARE) nephrometry system in predicting surgical outcomes after partial nephrectomy. BMC Urol. 2020;20 (1):146. doi:10.1186/s12894-020-00702-6

29. Porpiglia F, Amparore D, Checcucci E, et al. Current Use of three-dimensional model technology in urology: a road map for personalised surgical planning. Eur Urol Focus. 2018;4(5):652-656. doi:10.1016/j.euf.2018.09.012

30. Checcucci E, Amparore D, Pecoraro A, et al. 3D mixed reality holograms for preoperative surgical planning of nephron-sparing surgery: evaluation if surgeons'perception. Minerva Urol Nefrol. 2019.

31. Porpiglia F, Amparore D, Checcucci E, et al. Three-dimensional virtual imaging of renal tumours: a new tool to improve the accuracy of nephrometry scores. BJU Int. 2019;124(6):945-954. doi:10.1111/ bju. 14894
Research and Reports in Urology

\section{Publish your work in this journal}

Research and Reports in Urology is an international, peer-reviewed, open access journal publishing original research, reports, editorials, reviews and commentaries on all aspects of adult and pediatric urology in the clinic and laboratory including the following topics: Pathology, pathophysiology of urological disease; Investigation and treatment of urological disease; Pharmacology of drugs used for the treatment of urological disease. The manuscript management system is completely online and includes a very quick and fair peer-review system, which is all easy to use. Visit http://www.dovepress.com/ testimonials.php to read real quotes from published authors. 